\title{
The relation between proximity and brightness similarity in dot patterns
}

\author{
STEVEN W. ZUCKER \\ McGill University, Montreal, Quebec, Canada \\ KENT A. STEVENS \\ Massachusetts Institute of Technology, Cambridge, Massachusetts \\ and \\ PETER SANDER \\ McGill University, Montreal, Quebec, Canada
}

\begin{abstract}
The Gestalt studies demonstrated the tendency to visually organize dots on the basis of similarity, proximity, and global properties such as closure, good continuation, and symmetry. The particular organization imposed on a collection of dots is thus determined by many factors, some local, some global. We discuss computational reasons for expecting the initial stages of grouping to be achieved by processes with purely local support. In the case of dot patterns, the expectation is that neighboring dots are grouped as a function of proximity and similarity of contrast, by processes that are independent of the overall organization and the various global factors. We describe experiments that suggest a purely local relationship between proximity and brightness similarity in perceptual grouping.
\end{abstract}

The notion of grouping was introduced by Wertheimer $(1923 / 1958)$ to describe visual processes that take a collection of discrete elements, such as dots, and produce a more global one, such as a line. His argument was supported primarily by convincing visual demonstrations, like the one in Figure 1a, and culminated in a series of principles postulated to underlie visual organization (see, also, Koffka, 1935; Kohler, 1929). Central among these principles were grouping by proximity and grouping by similarity. Figure la demonstrates the proximity principle: the vertical spacing between the dots is less than the horizontal spacing, and hence, the dots appear to be grouped into vertical lines. Similarity among (or between) dots also influences the perceived organization: if the dot spacings are equal in the horizontal and vertical directions, but the dots in alternating

This report describes research done at the Computer Vision and Robotics Laboratory, McGill University, and at the Artificial Intelligence Laboratory, Massachusetts Institute of Technology. Research at McGill University was supported by NSERC Grant A4470; the research at M.I.T. was supported by AFOSR and NSF Grant 79-23110MCS. The authors thank P. Lamoureux for programming assistance and $\mathbf{P}$. Cavanagh for calibrating the display monitor. An earlier version of this paper appeared as M.I.T. AI Memo 670. Kent Stevens is now at the Department of Computer and Information Science, University of Oregon, Eugene, Oregon 97403. S. W. Zucker's and P. Sander's mailing address is: Computer Vision and Robotics Laboratory, Department of Electrical Engineering, McGill University, Montreal, Quebec H3A 2A7, Canada. rows are displayed at different intensities, one sees horizontal lines. Since the proximity and similarity principles are independent, they can be brought into "conflict" with one another, with proximity suggesting one organization (say vertical) and similarity another. Our plan was to use these conflicts to provide data for constraining future process-oriented theories of grouping.

There are many different approaches to theorizing about grouping processes. Most abstractly, these approaches can be distinguished by whether they are single- or multiple-process theories, and by whether they have local or global data support. In particular, rectangular grid patterns are highly regular and symmetric. These are both global properties of patterns, in that they hold over the entire pattern. They should be contrasted with the proximity and similarity relations over spatially neighboring dots that were discussed above, as these are local properties. Do all these factors, proximity, similarity, regularity, symmetry, and others, local and global, simultaneously combine within a single perceptual process to determine groupings, or is there a succession of processes: local processes that impose groupings, say, between dot pairs, and subsequent processes that build more global organizations out of the local groupings? The end result in either case is an organization among dots that is influenced by many factors; the difference lies in how the factors are applied and how the organization is constructed. There are several rea- 


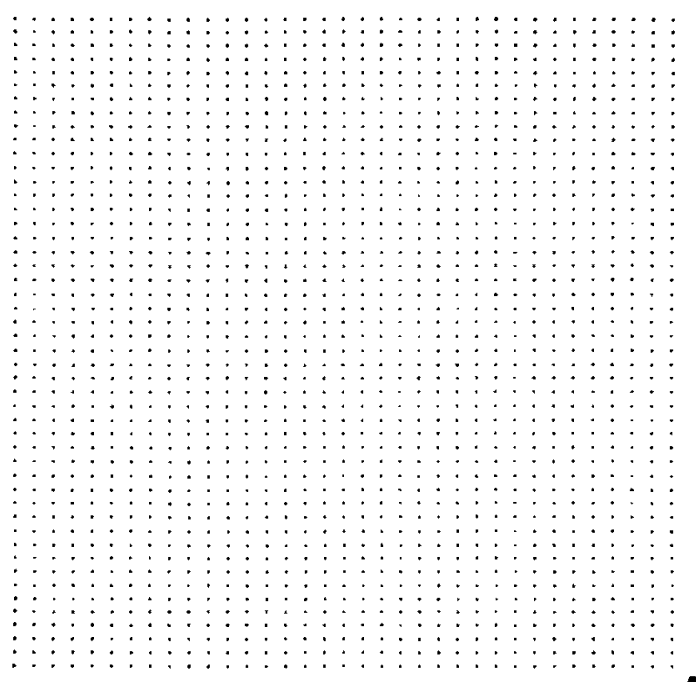

A

B

C D
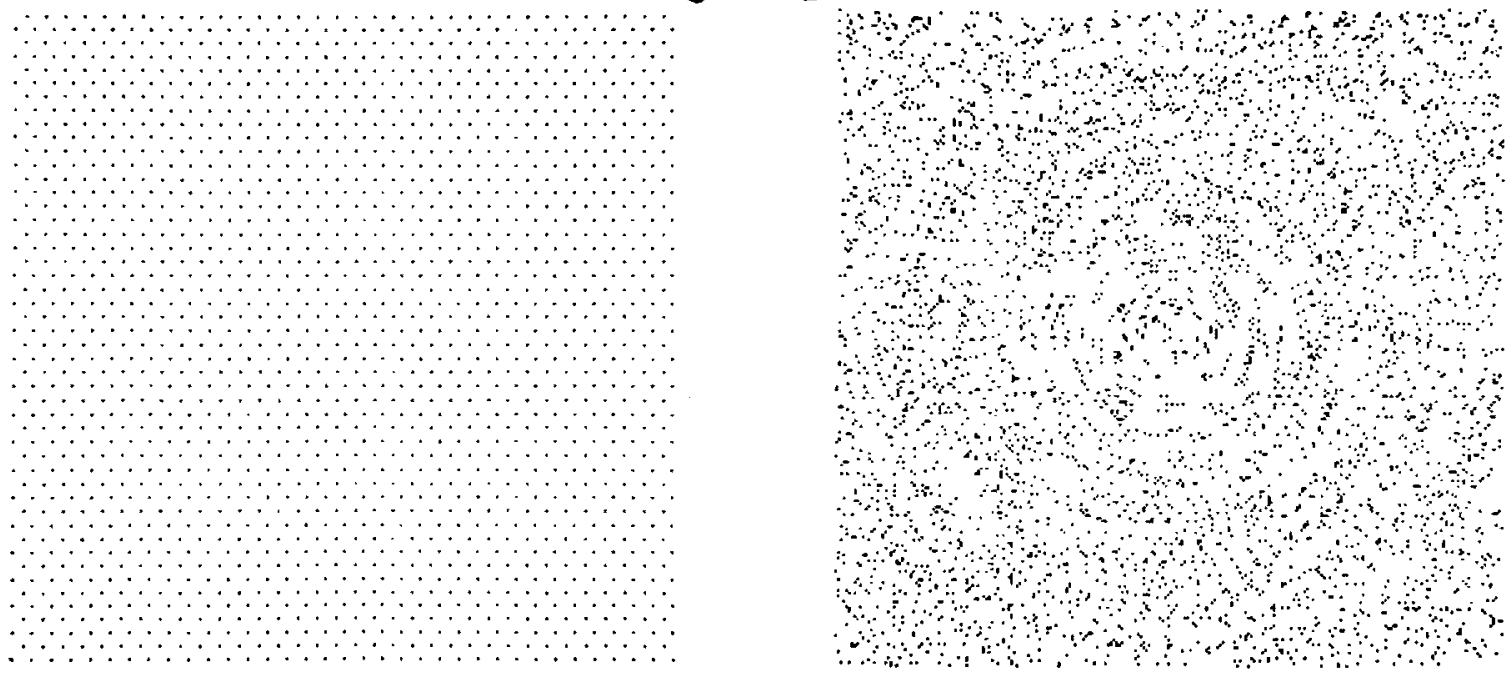

Figure 1. The dot patterns used to examine the role of contrast similarity and proximity in one-dimensional grouping. (A) A vertical organization, (B) a horizontal one, (C) a "hash" organization, and (D) a circular random dot pattern (see text for description).

sons, which we will discuss, for expecting multiple processes in which the earlier stages are supported only by local patterns of dots.

The empirical side of our argument is based on similarities in the psychophysical data obtained from three very different types of dot patterns. Each pattern is made from two subpopulations of dots, and in the experiments we varied the intensity (or another property) of one subpopulation. The first pattern is a rectangular grid that appears organized into columns when the contrast of both populations of dots is similar (Figure 1a) or into rows when they are of very different contrast (Figure $1 \mathrm{~b}$, where one population has zero contrast relative to the background to emphasize the alternative organization). The second pattern is a variant that, depending on the contrasts of the two populations of dots, yields two very different apparent organizations: a horizontal/vertical grid or a diagonal grid (Figure 1c). The third pattern is a circular random-dot moiré pattern (RDMP) (Figure 1d), created by displaying a pattern of random dots and then superimposing a copy of these random dots rotated by a small amount (see Glass, 1969, 1979).

There are three fundamental differences between the patterns selected as stimuli here. The first difference is orientation-Figures $1 \mathrm{a}$ and $1 \mathrm{~b}$ are vertical/ horizontal, while Figure 1c is horizontal/diagonal. 
Given the power of the oblique effect, such differences must always be taken into account. The second difference is in curvature-Figures $1 \mathrm{a}, 1 \mathrm{~b}$, and $1 \mathrm{c}$ contain straight lines, while Figure 1d contains circles. The third difference is a more subtle one. It centers on the spatial continuity of the patterns and the resultant percepts. The regular arrangements in Figures $1 \mathrm{a}, 1 \mathrm{~b}$, and $1 \mathrm{c}$, like the arrangements studied by the Gestalt psychologists, yield percepts that are continuously supported everywhere; the long lines are given by precisely, and evenly, spaced dots. The RDMP in Figure 1d, however, contains no such long, perfect curves. Rather, it contains more of a random arrangement resulting in an overall impression of "flow" rather than well-defined contours. Zucker (1983, Note 1) discusses these structural differences in much greater detail, as well as corresponding differences in the physical scene configurations from which they might arise.

Our principle psychophysical result is that, despite these differences, the three classes of patterns evoke very similar quantitative behavior with respect to contrast and proximity. On the basis of these similarities, then, it seems logical to infer common functional principles within the processes responsible for accomplishing them. But strong differences in the structure of the patterns considered further suggest that some variation may be required among these processes. If, however, a formulation could be found in which the entire process could be decomposed into stages, then the earlier stages could deal with the local variations in the patterns, and the later stages could be more global and, perhaps, common. One such decomposition, based on the differential geometry of curves, is presented in the next section (see also Zucker, Note 1). It is nicely consistent with other activities believed to be at the base of early visual information processing. The alternatives of global processes in the earlier stages, or of a plethora of singlestage, global processes, are much less natural and somewhat difficult to reconcile with the known neurophysiology.

\section{THEORETICAL DISCUSSION}

Why does the visual system, when presented with a set of discrete dots, tend to organize into contours those dots that are adjacent, similar, and (roughly) collinear? The answer to this question comes, we believe, from an examination of the role that continuous intensity contours play in vision: they signal physical discontinuities of various kinds, such as illumination or reflectance discontinuities, physical creases, or surface boundaries. An intensity discontinuity on the retina is signaled by collections of cortical neurones organized into local operators such as those modeled by Marr and Hildreth (1980). For a variety of reasons, however, neither this process nor any like it will immediately yield a globally contiguous contour. The standard reason is that the local operator responses are confounded by several factors, including occlusions of objects by foreground details, insufficient contrast in places, and various transducer artifacts. Consequently, rather than producing responses along a continuous contour path they result in a pattern of contiguous fragments and occasional gaps (see Marr \& Hildreth, 1980, Figure $8 \mathrm{~d}$ ). Some additional processing is clearly necessary for completing these fragments, that is, for grouping them into a complete contour. Gestalt similarity notions are seen to be related when one considers that the contour fragments arise from the images of coherent physical objects. That is, pieces of a physical contour project to fragments that are generally similar in contrast, intensity, and motion (if any) and, furthermore, are proximate and (locally) roughly collinear. These properties can then be used as local evidence for reconstructing the whole contour from its discontinuous fragments (Marr, 1982; Zucker, 1981). (Note that a form of this argument would hold for any local contour-detection operator.)

There is a more basic point, however, that comes not from a consideration of what is being measured, but, rather, from the nature of the measurement process itself. Each of the operators provides a single summary measurement over its receptive field (or, more precisely, its image support). Together, they yield an array of separate measurements, each of which is an isolated event associated with a point location. Somehow these pointwise events must be interpreted, or grouped, into more spatially extensive and more abstract structures (or, more precisely, into their representations). The inference of a "curve" connecting them is perhaps the simplest example of such a grouping process. Note that it is a constructive act, because the language of curves is drastically different from the language of points.

Inferring curves through pointwise measurements is relatively straightforward when the responses are dense and strong, but becomes more complex as the responses become ambiguous. Such ambiguities could arise when the dotted curves are dense or intersecting, when the responses are weak, or when the points are sparse. Dot patterns provide a particularly useful tool for studying the inference of such contours because they readily permit a separation of the geometry, or positioning, of the dots from the measurement values, or intensities. They can, furthermore, be viewed as a limiting case of operator responses because, as the operators' receptive fields increase and their overlaps decrease, the result is a dotted "response contour."

One approach to a class of computational theories that could support the inference of curves from dot arrays separates mathematically into two stages. Based on the differential geometry of curves, the goal 
of the first stage is to produce the "tangent" to the curve at a particular point (Caelli, Preston, \& Howell, 1978; Zucker, Note 1). This tangent indicates the direction in which the curve is moving as it passes through that point. It is in the second stage that curves are actually found which are consistent with these tangents. Note that there is a natural sense in which the first stage is "local" and the second more "global." Other constraints, such as the density of the dots, matter as well (see Zucker, 1983, Note 1, for further discussion of these constraints and a model applicable when the dots are "dense"; Stevens, 1978, deals with "sparse" distributions).

Psychophysical research on dot grouping has concentrated primarily on two-dimensional texture discrimination, wherein a dot pattern segregates into regions delimited by boundaries across which certain statistics vary discontinuously (Beck, 1966; Julesz, 1962, 1971; Olson \& Attneave, 1970). Subjectively, there is cohesion among similar texture elements and segregation between dissimilar elements. A common expectation is that the perceptual segmentation of texture arises by two-dimensional aggregation of similar texture elements; the similar elements cohere into demarcated regions. From this point of view, it is conceivable that the aggregation of dots into onedimensional chains is but a limited case of more general similarity grouping, particularly in view of the comparable role of brightness and proximity in each case (see Hochberg \& Silverstein, 1956; Prytulak \& Brodie, 1975; Rock \& Brosgole, 1964). But one cannot conclude from this analogy that the empirical results regarding the two-dimensional task apply equally to the one-dimensional task. Dot contours exhibit several perceptual differences from texture boundaries (e.g., regions differing in dot density) (Stevens, Note 2). Furthermore, the two-dimensional computation has both "similarity" and "dissimilarity" components, only the latter of which has any connection with the one-dimensional task (Zucker \& Hummel, 1979). It is the case, however, that (at least in principle) dot clustering can also be separated into local followed by global processing.

In summary, we have argued that the grouping of dots proceeds from local to more global processing, with the earlier stages defined to find such entities as "tangents at a point" and the later stages finding curves through these points. Since dots are rather unnatural stimuli, it was further argued that the dot grouping machinery was (at least) analogous to that used for intensity-discontinuity detection. One consequence of even loosely specifying mechanisms in this fashion is that further constraints on the processing start to emerge. In particular, the process of finding tangents readily separates into two further conceptual stages: (1) the measurement of certain properties of the dot arrays, and (2) the determination of which of these measurements are indicative of dotted con- tours, and which are artifactual (e.g., those perturbations of the measurements due to noise, closely spaced curves, weak responses, etc.). It follows, in particular, that, for weak responses, the second of these stages should be more difficult, since it amounts to a decision process based on data with a lower signal/noise ratio. The possibility of more psychophysical variation should be expected under these conditions, which is exactly what we have found for all three dot-pattern classes. These data have independent interest as well, because they demonstrate an interaction between the geometry (or location) of dots and their contrast that must be accounted for by any viably complete theory of grouping.

\section{EXPERIMENT}

The purpose of the experiment was to determine the relationship between proximity and similarity (of intensity) in the grouping of dots for various dot patterns. The basic experimental paradigm was an extension of that implicit in the Gestalt demonstrations. Subjects viewed brief displays and were forced to state either the perceived grouping or that none was present. Each experimental pattern was designed to have two very different apparent organizations, such as the vertical versus horizontal in Figure 1a, which allowed a simple forced-choice protocol. (If neither was seen, then the subject could respond 'nil'). Our objective was to quantify the strength of the perceptual grouping into one of the two apparent dot organizations as the similarity of neighboring dots was systematically varied. Spacing between dots and intensity of both the dots and the background were varied.

\section{Method}

Subjects. There were eight subjects, five males and three females. Two had had experience with the experimental tasks and were aware of the goals; the rest were naive in both regards. All had normal or corrected vision. Not all subjects were presented with every pattern (selection was based on availability).

Apparatus. The stimulus images were generated by a Grinnell GMR-27 graphic display processor on a DEC VAX 11/780 computer and displayed on a Tektronix 670A-1 color monitor. The experiments were conducted in a dimly illuminated room, with a background reflectance of $0.58 \mathrm{~cd} / \mathrm{m}^{2}$ from the monitor. The subject sat on a chair $2 \mathrm{~m}$ from the monitor and, although instructed to sit still, was permitted slight movement.

Procedure. The experiment consisted of several sessions. During each session, the subject was presented with a sequence of images that were constant in dot proximity but variable in similarity. Each image was flashed for $250 \mathrm{msec}$. The subject viewed a uniform gray display between stimulus flashes, of $133 \mathrm{~cd} / \mathrm{m}^{2}$ chosen to be intermediate between the calibrated minimum $\left(0.58 \mathrm{~cd} / \mathrm{m}^{2}\right)$ and maximum $\left(267 \mathrm{~cd} / \mathrm{m}^{2}\right)$ intensities. Postmasking (for instance by a random-dot pattern) was considered unnecessary for this task. For each stimulus presentation, the subject responded with one of three answers; for example, for the patterns of Figure 1a, the choice was between "horizontal" and "vertical," depending on perceived linear orientation, and "nil" when no dots could be distinguished (this occurred when they were all at one 
intensity with the background). The subject's responses were recorded and later plotted as the accompanying graphs.

To become familiar with the task protocol, each subject was given a trial run of 10 images before data collection was begun. All subjects used in the experiment grasped the procedure within these first 10 trials.

Stimuli. Three types of dot pattern were used as stimuli: (1) those with a horizontal or vertical orientation (Figures $1 \mathrm{a}$ and 1b); (2) those organized in either a horizontal/vertical direction or a diagonal direction (which we refer to as "hash" patterns, (Figure 1c); and (3) rotated RDMPs (Figure 1d). Each type of pattern consisted of two overlapping populations of dots; one was fixed in intensity, and the other varied across trials. All dots subtended $1.2 \mathrm{~min}$ of arc. Two apparent organizations were possible from each presentation, depending on whether the two dot intensities, one for each population, were seen as similar or dissimilar. One set of dots was held fixed at the maximum intensity, while the other took on one of five values varying from the minimal to the maximal screen intensities. The background intensity was varied over five values, so that the effect of dot contrast with respect to the background could be assessed as well. Twenty-five intensity combinations were thus possible, each of which was shown five times in random order. The result was a total of 125 presentations for each pattern type per session.

In certain combinations, some or all of the dots were indistinguishable from the background. For example, in one trial, all of the dots and the background were at equal intensities. Such trials were controls for the subject-he had to respond "nil." The stimulus patterns will now be described in more detail.

Horizontal/vertical. A typical image from the horizontal/vertical discriminations is given in Figure 1a; it is also presented schematically in Figure $2 a$. Here the xs represent dots with intensity fixed at a maximum over all images of a sequence, and the os represent dots with variable intensity. All of the os were the same intensity for each trial, and varied between trials, so that the $x$ and $o$ dots could have the same or different contrasts with respect to the background. The distance between the horizontal xs was $10 \mathrm{~min}$ of visual angle; the distance between vertical xs was $13 \mathrm{~min}$; and the os were intermediate between the vertical xs. Thus, with all dots at similar intensity and substantially above background, the pattern appears vertical, as in Figure 1a; with the os at background intensity, the pattern appears horizontal, as in Figure 1b.

The three responses for these images were "horizontal," "vertical," and "nil," depending on the predominant orientation (if any) in the pattern. To compensate for any bias toward horizontal or vertical, the patterns were presented as in the above figure or rotated by 90 deg (each orientation had probability .5). The same patterns were presented in later sessions in which all dot spacings were doubled.

Linear/hash. The linear/hash figures are similar to the vertical/ horizontal. An example is given in Figure 1c and shown schematically in Figure $2 \mathrm{~b}$, where the xs represent dots at fixed maximum intensity and the os are dots at variable intensity. The spacing between the horizontal xs was $10 \mathrm{~min}$ and that between the

$$
\begin{array}{lllllll}
x & x & x & x & x & x & x \\
0 & 0 & 0 & 0 & 0 & 0 & 0 \\
x & x & x & x & x & x & x \\
0 & 0 & 0 & 0 & 0 & 0 & 0 \\
x & x & x & x & x & x & x \\
0 & 0 & 0 & 0 & 0 & 0 & 0 \\
x & x & x & x & x & x & x
\end{array}
$$

\section{A}

Figure 2. Schematic diagram where xs are dots of maximum in tensity and os are dots of variable intensity. vertical $\mathrm{xs}, 13 \mathrm{~min}$. The os were intermediate between the $\mathrm{xs}$ in both directions, giving a spacing between $\mathrm{xs}$ and os of $8.2 \mathrm{~min}$.

The choice of responses for these patterns was between "linear" (when either horizontal or vertical organization was seen), "hash" (when a grid-like or diagonal organization was seen), and "nil" (when no dots were seen).

Half the images for each type of pattern were presented as above, and half were rotated by $90 \mathrm{deg}$. The same patterns were presented later at twice the scale. Intensities of os and backgrounds were varied as in the horizontal/vertical.

Random-dot moiré patterns. The images presented in these experiments were rotational RDMPs, similar to that in Figure 1d. They were created by rotating a pattern of 1,000 randomly generated dots by $5 \mathrm{deg}$ and displaying the original and transformed dot patterns together. The original dots were at a fixed intensity over all the images of a stimulus sequence, and the intensities of both the transformed dots and the background were variable. The responses were chosen from among "grouped" (for a circular organization), "ungrouped," and "nil." Depending on the experimental parameters, the original and rotated sets of dot sets would (1) group together into one organization, (2) remain separated into two sets with a different organization, or (3) blend into the background. In the case of RDMPs, (1) corresponds to the circular percept, (2) to a random pattern, and (3) to the test condition in which the dots are indistinguishable from the background.

\section{Results and Discussion}

Our results are shown as graphs, in which the proportion of grouping is plotted as a function of both the intensities of the background and of the variableintensity dots. All graphs are to the same scale, with the maximum indicating that five such groupings were reported in the five presentations of that combination of variable-dot and background intensities. (In this case, the fixed and the variable dots would have grouped completely.)

Figure 3a shows a typical result for one subject in the vertical/horizontal task. For this experiment, the fixed dots were at maximum screen intensity $\left(267 \mathrm{~cd} / \mathrm{m}^{2}\right)$. The graph can be read as follows. For each value of the background, a curve is specified that shows the intensities of the variable dots that grouped with the fixed ones. Running in the orthogonal direction are curves corresponding to fixed values for the variable dots; they show the variation with changes in the background level. Taken together, the two families of curves specify grouping as a function of both of these variables. The presence of the peaks completely on one side of the diagonal indicates that the subject never grouped dots with opposite contrast (relative to the background). As the background became lighter, the variable dots had to be lighter before they grouped with the fixed dots and defined a new organization. Note, furthermore, that, for this intensity quantization, about one step difference from the background was sufficient for grouping, with a bit more for the lower values of intensity.

This result is typical of those obtained for all three tasks. In Figure 3b, we show the mean for six subjects, with the standard deviation between them in Figure 3c, for the vertical/horizontal task. In Fig- 


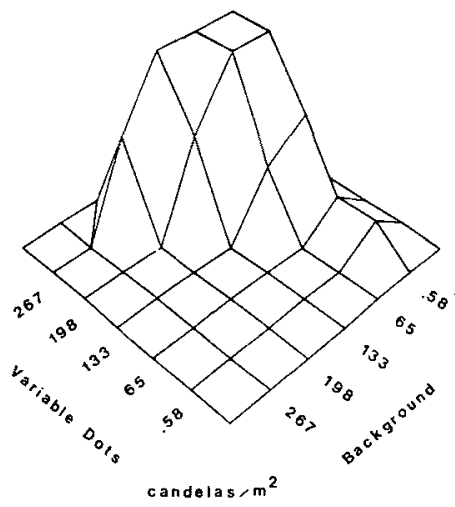

A

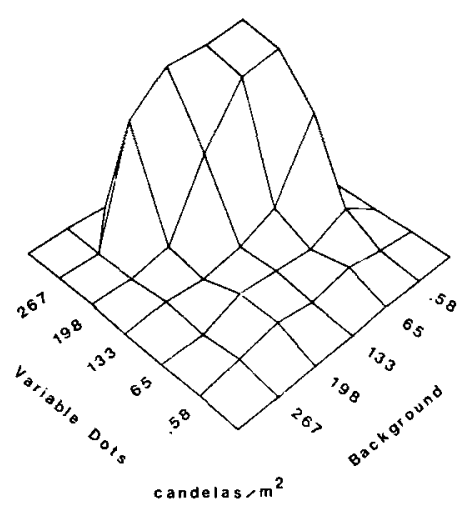

B

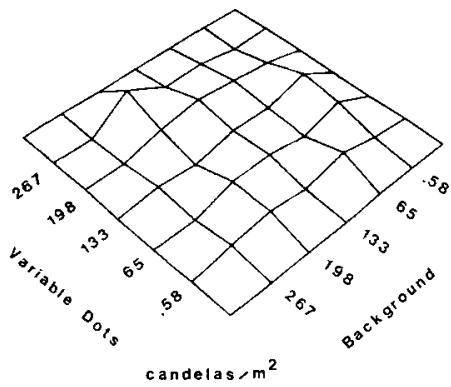

C

Figure 3. Results of the vertical/horizontal task. (A) A typical result for one subject. The two axes denote in tensity scales for the variable dots and for the background. Height is proportional to the percentage of grouping between the variable and fixed dots. There were five trials for each combination of background and variable dot in tensity. (B) The mean performance and $(C)$ the standard deviation uver six subjects, five trials for each subject.

ure 4a, we present a typical result from the linear/hash task, with the mean and standard deviations for four subjects in Figures $4 b$ and $4 c$. This task showed more variation than the vertical/horizontal, and the subjects on occasion reported that they had found it harder to decide between the two organizations (there being a wider range over which either organization could be seen). The third task, which involved RDMPs, was the easiest to perform and shows the "cleanest" result (see Figure 5a for a typical response and Figures $5 \mathrm{~b}$ and $5 \mathrm{c}$ for statistics over 3 representative subjects. In each case, dots with similar contrast (relative to the background) group together, provided they are readily distinguishable from the background. Note, from the graphs, that grouping requires less intensity difference (between fixed and variable dots) at the higher intensities of the background than at the lower for both the vertical/horizontal and the linear/hash tasks. These findings continued in subsequent experiments, and we take them as supporting the distinction made at the end of the previous section that the decision process involved in interpreting the measurements is more uncertain in the "weaker" situations. There is, of course, no symmetric difficulty at the high intensity, or "strong" end of the curve.

The next series of experiments were repetitions of the vertical/horizontal task, with twice the spacing between dots (20 and $13 \mathrm{~min}$ rather than 10 and $6.5 \mathrm{~min})$. Again, the fixed-intensity dots were constant at $267 \mathrm{~cd} / \mathrm{m}^{2}$. The data are qualitatively very similar; see Figures $6 \mathrm{a}$ and $6 \mathrm{~b}$ for the statistics over three subjects. It is worth noting that the task is much more difficult to perform at the longer interdot spacings. The impression of dotted lines, both vertically and horizontally, is much less vivid, and the task judgment seems to be made on the basis of shorter dotted segments, or sometimes even mere pairings. Doubling the spacings again emphasizes these perceptual differences from the original, closely spaced

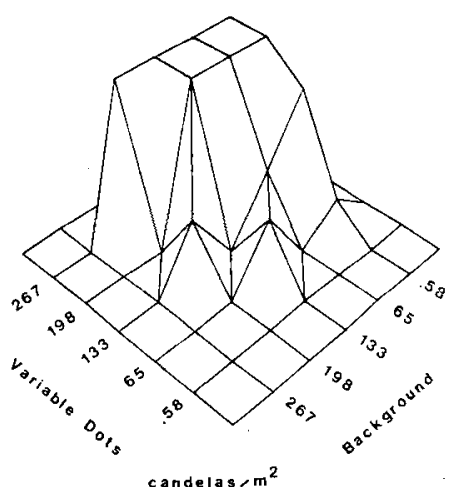

A

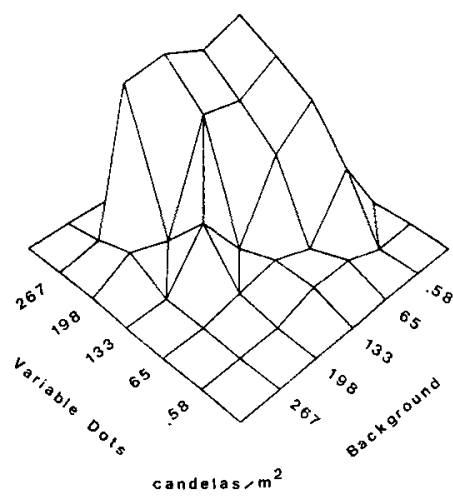

$\mathbf{B}$

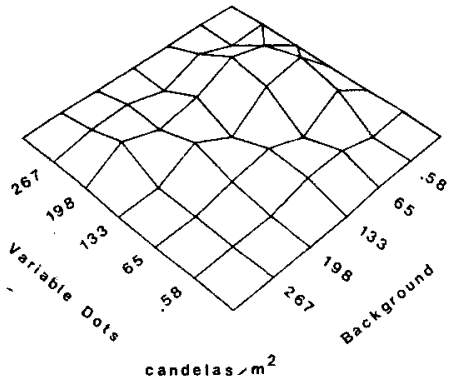

C

Figure 4. Results of the linear/hash task. (A) The result for one subject, and (B) the mean and (C) the standard deviation for four subjects. Note that this task was much less clear than either the vertical/horizontal or the RDMP pattern task. 


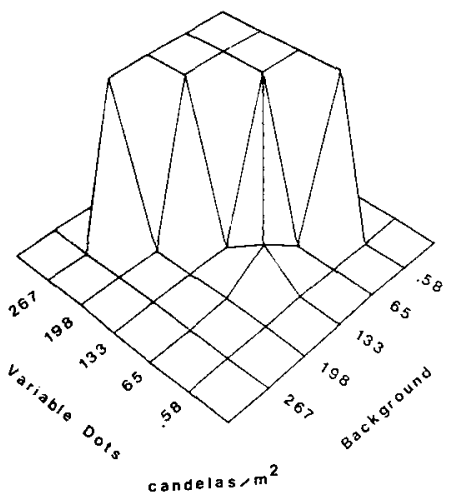

A

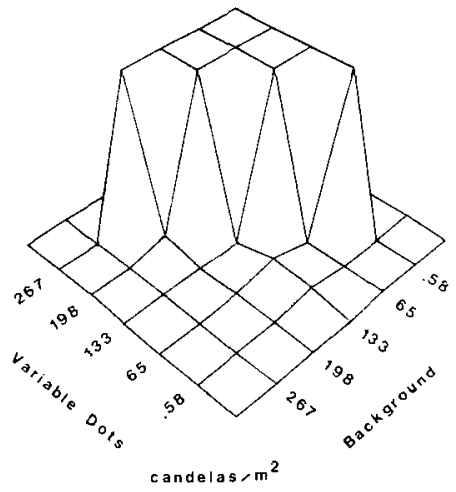

B

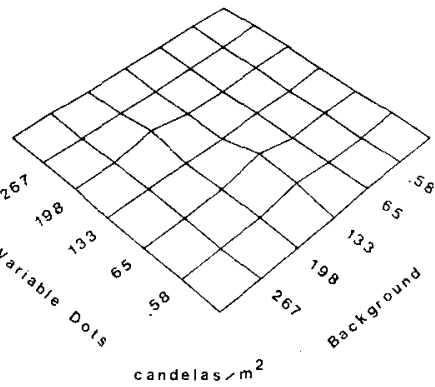

C

Figure 5. Results of the RDMP task. (A) Result for one subject, and (B) the mean and (C) the standard deviation for three subjects. The sharp "corners" in the above graphs indicate that this task was the easiest to perform; as long as the variable dots were of the same contrast as the fixed dots, the groupings formed.

task, ${ }^{1}$ with the result that, for two subjects, dotted lines were clearly seen only for intensity combinations with the background dark and the variable dots light (within two quantization steps of the fixed, bright dots). But the task could still be performed with results like those in Figure 6.

The tasks described above were conducted with the fixed dots always at the maximum screen intensity. To show that the results hold with variations in this intensity, the experimental tasks were repeated with intensity set to an intermediate gray level. Now it became possible for the dots to group on both sides of the background, or, in terms of the graphs, on both sides of the diagonal. The result is much more complicated, with the peaks previously confined to one side now present on both sides. The results for one subject performing the RDMP task (with the fixed dots at $133 \mathrm{~cd} / \mathrm{m}^{2}$ ) are shown in Figure $7 \mathrm{a}$; the statistics for three subjects' performance on all three tasks at this intensity level are given in Figures $7 b$ and $7 \mathrm{c}$. Note that the variance is now a bit higher, but that the curves are very similar. Finally, in Fig- ures $8 \mathrm{a}$ and $8 \mathrm{~b}$, we show the results from one subject performing the three tasks with the fixed dots at the minimum intensity $\left(0.58 \mathrm{~cd} / \mathrm{m}^{2}\right)$. It is essentially the "mirror" image of the original-task results (cf. Figure 3). It is especially interesting that, as in the original task, the slope is steep when the variable dots approach the fixed dots in intensity, but is more gradual (over two quantization steps) when they are at opposite ends of the scale.

Since the RDMP task provided the least variance, we chose it as the task for a closer examination of the effect of intensity on grouping in the range in which the transition occurs. The original experiment was repeated with a much smaller range of intensity variation between the fixed and variable dots. The results for two subjects are shown in Figures 9a and $9 \mathrm{~b}$. The background was set to $133 \mathrm{~cd} / \mathrm{m}^{2}$, and the fixed dots were at maximum intensity. The transition is rather abrupt.

In summarizing these data, we feel that a real pattern has emerged. It is illustrated in Figures 10a and $10 \mathrm{~b}$, which show the statistics over all three tasks,

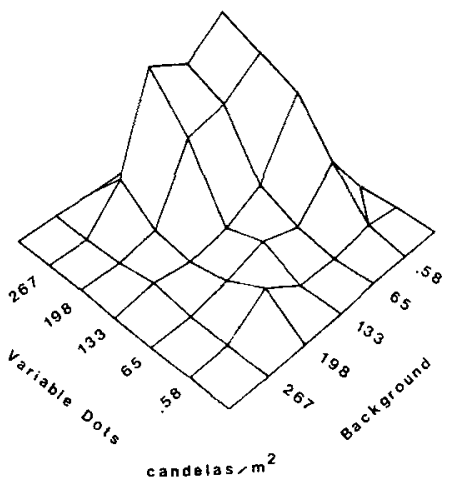

A

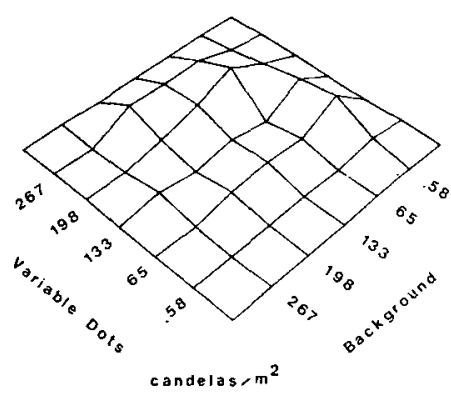

B

Figure 6. Repeat of the vertical/horizontal task with twice the spacing between dots. (A) The mean and (B) the standard deviation for three subjects. Note that the results here are much noisier than for the more closely spaced tasks. 


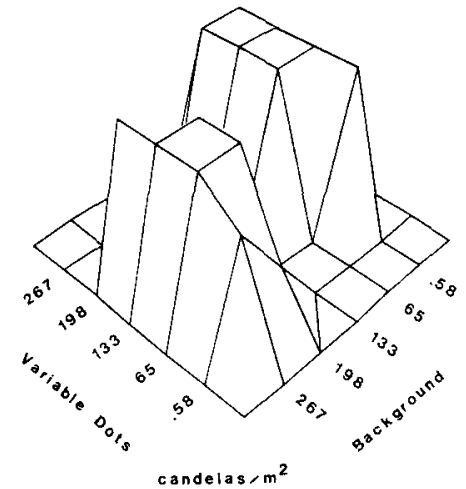

A

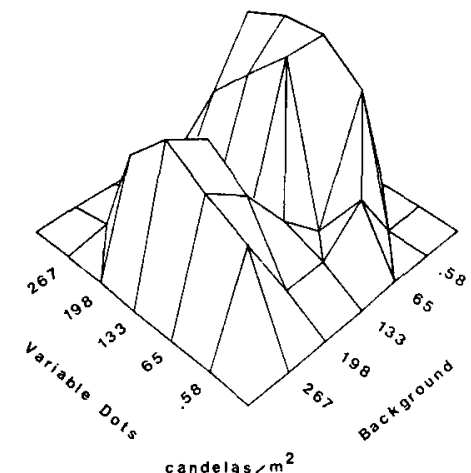

B

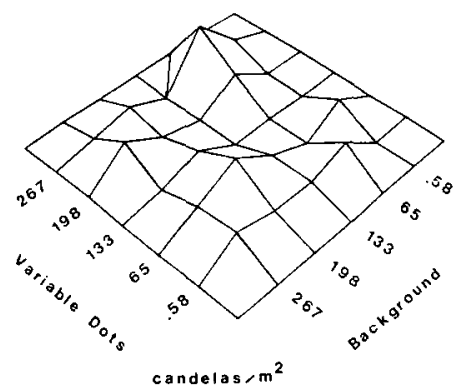

C

Figure 7. Results of the RDMP task with fixed dots at intermediate gray level $\left(133 \mathrm{~cd} / \mathrm{m}^{2}\right)$ and dots of same or opposite contrast sign relative to background. It now becomes possible to form two separate groupings, one when the dots are brighter than the background and the other when they are darker. Hence, there are two peaks in the graph, one on either side of the diagonal. (A) the results of one subject, and (B) the mean and (C) the standard deviation for three subjects.

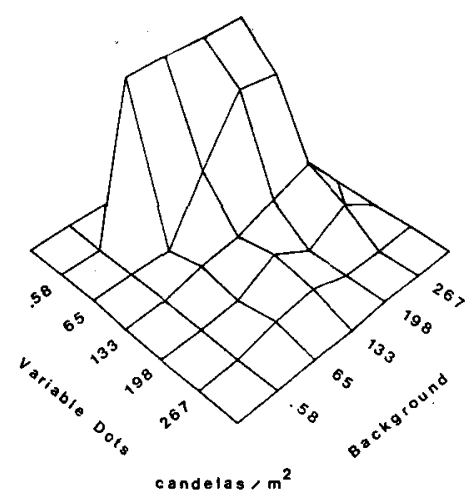

A

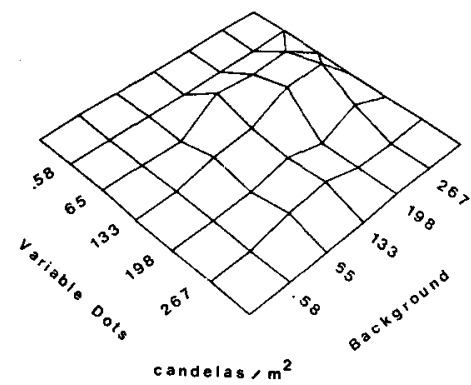

B

Figure 8. Horizontal/vertical task (see Figure 3) but with fixed dots at $0.58 \mathrm{~cd} / \mathrm{m}^{2}$. Since the fixed dots are now dark, the result is symmetrically the same as that in Figure 3.

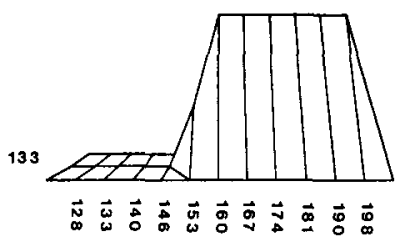

Variable Dots
candelas $-\mathrm{m}^{2}$

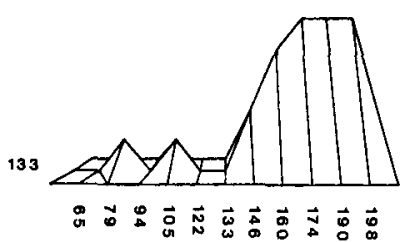

Variable Dote

candelas $/ \mathrm{m}^{2}$

B

Figure 9. Repeat of RDMP task with smaller range of intensity variation between the fixed and the variable dots. Results for two subjects are shown, one in $A$ and the other in B. The slope is rather sharp in both cases. 


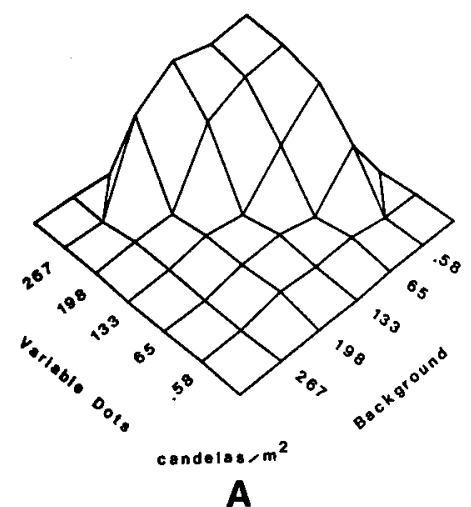

A

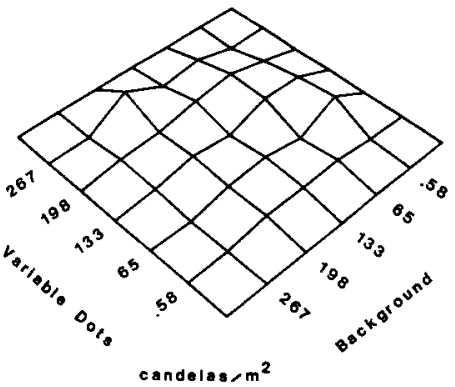

B

Figure 10. Statistics across all three tasks, subjects, and trials; (A) mean and (B) standard deviation.

all subjects, and all trials (with the fixed dots at maximal intensity and the small spacing between them). Dots virtually never group across the background, but readily group when they differ from it. That is, similar contrast leads to grouping, opposite (even small) contrast destroys it. This observation generalizes one made by Glass and Switkes (1976) regarding RDMPs containing dots of opposite contrast (see also Julesz, 1965). The variability occurs when the dots are close to the background, as indicated by the way in which the two "tails" of the peak slope gradually down to zero. It is also shown in the standard deviation plot of Figure 10b, which is highest at these two places. This summary graph, however, obscures the individual task differences discussed previously.

It is our belief that these results reflect early information processing more than mere cognitive judgments. Two arguments support this belief, both of which are based on trying to alter the results by changing the data. First, presuppositions about the patterns do not seem to matter. Second, ${ }^{2}$ if the subject is asked to keep the dots segmented according to intensity and just to group similar-intensity dots, the phenomenology changes drastically. The subject must try to remember the brief pattern for a more selective analysis. This takes much longer to do than the original task, and is often extremely difficult or impossible. The immediately available precept already appears grouped.

\section{Further experiments}

All of the tokens in these grouping tasks have been dots, and the question immediately arises as to whether small variations in these tokens would still permit grouping. We attempted two such variations-the variable dots were made larger, by a factor of two (so that they were no longer pinpoints), and were grown into short line segments by increasing their length by a factor of three. An example of the latter patterns is shown in Figure 11. It immediately became clear, however, that when these new images were substituted into our experimental protocol for the vertical/horizontal or linear/hash tasks, the new tokens always grouped separately from the original dots. Thus, the visual system is very sensitive to differences of "type," apparently more so than to small intensity changes, and these differences predominate over intensity differences for these two tasks. Subjects could "cognitively" report the groupings, and could decide whether or not to group dots with lines, but the immediate perceptual effect that is so clear with dots is gone. Every subject reported this difference in the task, and no data were formally collected.

\section{CONCLUSIONS}

Grouping is a generic term for processes that take collections of perceptually distinct visual elements and form them into more global, and perhaps more abstract, wholes. It seems clear that there is a diver-

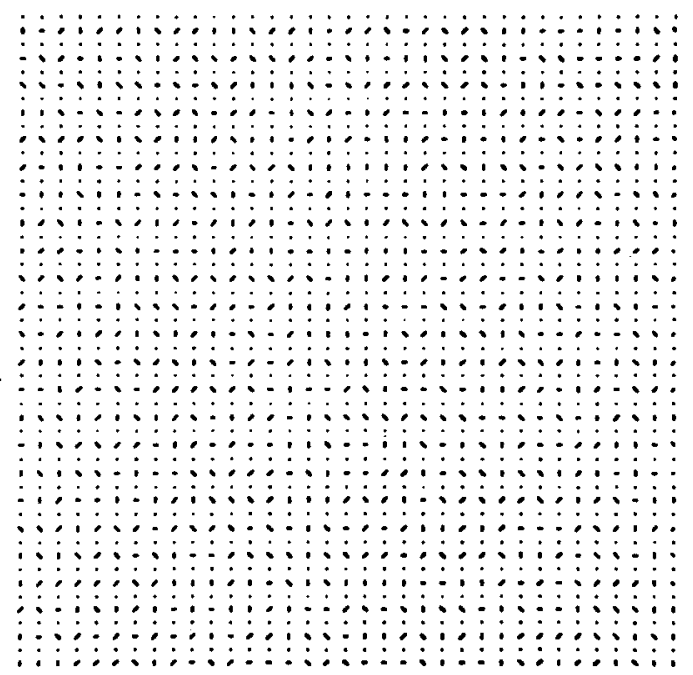

Figure 11. Example pattern combining dots and short line segments. When the line segments were more than 5 dots in length, they rarely grouped with the dots. 
sity of such processes, ranging from functions very early on in visual information processing, such as completion of intensity-discontinuity contours, to later processes involving surfaces. There may even be a cognitive component to grouping (Kanizsa, 1979). We argued in this article that the Gestalt demonstrations involving dots were exercising the earlier components of this system in a controllable manner. Hence, they are amenable to study, and that was the primary purpose of this article.

In particular, we studied the psychophysics of three dot-grouping tasks, in which spacing, intensity, and shape parameters were varied. Subjects behaved very similarly for these three tasks, in that they always grouped dots that were immediately distinguishable from the background, provided they had similar contrast with respect to that background.

These results suggest two principal conclusions. First, since the psychophysics are very similar across the different tasks, it seems likely that they result from the same kind of (if not the same) process. This could be the process(es) originally responsible for estimating orientation at a point. Second, since the global geometry of the contours is different in the various stimulus patterns, the processing would seem to be after a local quantity. Again, this could be a local orientation selectivity. Both of these conclusions are consistent with theoretical activities in early visual information processing, which were used to argue for the existence of a mechanism for contour completion. Such mechanisms would have both of the above properties.

\section{REFERENCE NOTES}

1. Zucker, S. Early orientation selection and grouping: Evidence for type I and type II processes (Technical Report 82-8). Montreal: McGill University, Department of Electrical Engineering, August 1982.

2. Stevens, K. A. Contours in dot patterns: Evidence and algorithms. Manuscript in preparation. (Available as M.I.T. A.I. Lab Memo 669, 1982.)

\section{REFERENCES}

BECK. J. Effect of orientation and shape similarity on perceptual grouping. Perception \& Psychophysics, 1966, 1, 300-302.

Caelli, T., Preston, G., \& Howell, E. Implications of spatial summation models for processes of contour perception: A geometric perspective. Vision Research, 1978, 18, 723-734.

GLA8s, L. Moiré effect from random dots. Nature, 1969, 223, 578-580.

GLAss, L. Physiological mechanisms for the perception of random dot moiré patterns. In H. Haken (Ed.), Pattern formation by dynamic systems and pattern recognition. Berlin: SpringerVerlag, 1979.

Glass, L., \& Switkes, E. Pattern recognition in humans: Correlations which cannot be perceived. Perception, 1976, 5, 67-72.

Hochbero, J., \& Silverstein, A. A quantitative index of similarity: Proximity versus difference in brightness. American Journal of Psychology, 1956, 69, 456-458.

JulEsz, B. Visual pattern discrimination. IRE Transactions on Information Theory, 1962, 8, 84-92.

Julesz, B. Texture and visual perception. Scientific American, $1965,212,38-54$

Julesz, B. Foundations of cyclopean perception. Chicago and London: University of Chicago Press, 1971.

Kanizsa, G. Organization in vision. New York: Praeger, 1979.

KoffKA, K. Principles of Gestalt psychology. New York: Harcourt, Brace, 1935.

KoHle R, W. Gestalt psychology. New York: Liveright, 1929.

MARR, D. Vision: A computational investigation into the human representation and processing of visual information. San Francisco: Freeman, 1982.

Marr, D., \& Hildreth, E. Theory of edge detection, Proceedings of the Royal Society, London, B, 1980, 207, 187-217.

Olson, R. K., \& Atrneave, F. What variables produce similarity grouping? American Journal of Psychology, 1970, 83, 1-21.

Prytulak, L. S., \& Brodie, D. A. Effect of length, density, and angle between arms on Gestalt grouping. British Journal of Psychology, 1975, 66, 91-99.

Rock, I., \& Brosoole, L. Grouping based on phenomenal proximity. Journal of Experimental Psychology, 1964, 67, 531-538.

Stevens, K. A. Computation of locally parallel structure. Biological Cybernetics, 1978, 29, 19-28.

Wertheimer, M. Untersuchungen zur Lehre von der Gestalt. Psychologische Forschung, 1923, 4, 301-350. [Abridged translation: Principles of perceptual organization. In D. C. Beardslee \& M. Wertheimer (Eds.), Reading in perception. New York: D. Van Nostrand, 1958.]

ZUCKER, S. Computer vision and human perception: An essay on the discovery of constraints. Proceedings of the Seventh International Joint Conference on Artificial Intelligence. Menlo Park, Calif: American Association for Artificial Intelligence, 1981.

ZUCKER, S. W. Cooperative grouping and early orientation selection. In O. Braddick \& A. Sleigh (Eds.), Physical and biological processing of images. New York: Springer, 1983.

Zucken, S. W., \& Hummel, R. A. Toward a low-level description of dot clusters: Labeling edge, interior, and noise points. Computer Graphics and Image Processing, 1979, 9, 213-233.

\section{NOTES}

1. Zucker (Note 1) discusses a size/density constraint active in dot grouping. It is rather precisely localized at about 4-5 dot diameters. That is, when the spacing between dots is less than 4 diameters, the dotted contour behaves very much like a solid contour; less dense spacings lead to somewhat different behaviors. Equipment limitations prevented us from conducting these experiments on the dense side of this constraint.

2 . This protocol was suggested by one of the reviewers.

(Manuscript received August 20, 1982; revision accepted for publication May 26, 1983.) 